\title{
Nova espécie de Pimelodus (Siluriformes, Pimelodidae) dos rios Tapajós e Tocantins, Brasil
}

\author{
Frank Raynner V. Ribeiro ${ }^{1,2}$ \& Carlos Alberto S. de Lucena ${ }^{1}$
}

\begin{abstract}
1. Laboratório de Ictiologia, Museu de Ciências e Tecnologia, Pontifícia Universidade Católica do Rio Grande do Sul, Av. Ipiranga, 6681, 90619-900 Porto Alegre, RS, Brasil. (fraynner@pucrs.br; lucena@pucrs.br)

2. Bolsista do CNPq, Curso de Pós-graduação em Zoologia, PUCRS.
\end{abstract}

\begin{abstract}
A new species of Pimelodus (Siluriformes, Pimelodidae) from Tapajós and Tocantins rivers, Brazil. Pimelodus tetramerus sp. nov. differs from the other species of Pimelodus Lacepède by its typical coloration, with four dark stripes on the sides of the body, the most dorsal beginning just behind the pre-dorsal plate and finishing at the end of the adipose-fin base; the second, wider than the first one, running just above the lateral line and extending from the lateral border of the supra-occipital process to the caudalfin base. Both are fragmented on its anterior portion, forming dark points that may join to form a vermiculate pattern. The dark dots and the vermiculate pattern extend over the dorsum, anterior to the dorsal-fin and head. The third stripe, also wide, runs just below the lateral line and begin near the upper region of the postcleithral process and extends until the caudal fin base. The fourth stripe, shorter than the three others, and absent in a few cases, begins above of the postcleithral process and ends nearly the pelvic fin. Moreover, the following are also diagnostic of the new species: presence a narrow light stripe on the lateral line, with about $1 / 3$ of the width of the third dark stripe, and a dark longitudinal bar on the ventral lobe of caudal fin. The new species shares the three separate trigeminofacial nerve foramina with others species of pimelodids.
\end{abstract}

KEYWORDS. New species, "mandi”, taxonomy, lower Amazonas river.

RESUMO. Pimelodus tetramerus sp. nov. difere das outras espécies de Pimelodus Lacepède por sua coloração típica, que consiste de quatro faixas escuras sobre os lados do corpo, a primeira, mais dorsal, iniciando logo após o final da placa pré-dorsal e terminando no final da base da nadadeira adiposa; a segunda, mais larga que a primeira, logo acima da linha lateral, percorre desde a borda lateral do processo supra-occipital até a base da nadadeira caudal. Ambas faixas estão fragmentadas em sua porção anterior, formando pontos escuros que, ocasionalmente, podem estar unidos, compondo um padrão vermiculado. Tais pontos escuros e/ou padrão vermiculado se distribui sobre a região pré-dorsal e cabeça. A terceira faixa, tão larga quanto a segunda, logo abaixo da linha lateral, inicia junto à região superior do processo pós-cleitral e estende-se até a base da nadadeira caudal e a quarta, de menor comprimento, podendo estar fraca ou ausente, iniciase logo abaixo do processo pós-cleitral e finaliza até próximo à nadadeira pélvica. Além disso, constituem caracteres diagnósticos da nova espécie a presença de uma estreita faixa clara sobre a linha lateral, com aproximadamente $1 / 3$ da largura da terceira faixa escura, e a presença de uma barra escura percorrendo longitudinalmente o lobo ventral da nadadeira caudal. A nova espécie compartilha o forâmen trigeminal tripartido com outras espécies de pimelodídeos.

PALAVRAS-CHAVE. Nova espécie, mandi, taxonomia, baixo rio Amazonas.

De PINNA (1998) propôs elevar as três subfamílias de Pimelodidae (Pimelodinae, Heptapterinae e Pseudopimelodinae) ao nível de família. Neste rearranjo, Pimelodidae corresponde à subfamília Pimelodinae de LUNDBERG et al. (1991b) e inclui Hypophthalmus Agassiz in Spix \& Agassiz, 1829, antes em Hypophthalmidae, e exclui um grande número de espécies que estão hoje agrupadas em Heptapteridae e Pseudopimelodidae. Esta proposta foi utilizada por SwARÇa et al. (2000) com Pimelodidae e Rhamdiidae, esta última correspondendo a atual Heptapteridae.

Pimelodidae agrupa atualmente 83 espécies, distribuídas em 30 gêneros. No entanto, estima-se que existam cerca de 45 espécies não-descritas (REIS et al., 2003). Seus representantes são exclusivos de água doce e se distribuem pela América do Sul e Panamá (LunDBERG \& LitTMANN, 2003).

O gênero Pimelodus Lacépède, 1803, aparentemente parafilético, possui um antigo e complexo histórico taxonômico, mesmo com suas espécies, antes distribuídas por quase todo o mundo, estando agora restritas à América do Sul (LundBerg \& LitTMAnN, 2003).

MeEs (1974) coloca sob o nome grupo-maculatus quatro espécies, $P$. maculatus Lacépède, 1803 , P. blochii Valenciennes, 1840, P. albofasciatus Mees, 1974 e $P$. clarias (Bloch, 1752), essa última podendo constituir-se em sinônimo de P. blochii. LundBerg et al. (1991b) identificaram um complexo caráter apomórfico de nervos craniais e forâmens trigeminofascial que diagnostica um grupo de espécies de Pimelodus, entre as quais encontrase $P$. maculatus, espécie-tipo do gênero, e $P$. blochii. Pimelodus albofasciatus não foi examinada por LUNDBERG et al. (1991b) quanto ao caráter proposto. Mais tarde, LUNDBERG \& PARISI (2002) ampliam o grupo (denominado aqui de grupo-maculatus), verificando a presença do caráter em Iheringichthys labrosus (Lütken, 1874), contrário a Vidal \& Lucena (1999), Bergiaria westermanni (Lütken, 1874) e Cheirocerus abuelo (Schultz, 1944).

Das 24 espécies válidas de Pimelodus, sete foram descritas ou assinaladas na bacia Amazônica: Pimelodus albofasciatus Mees, 1974; P. altissimus Eigenmann \& Pearson in Eigenmann \& Allen, 1942; P. blochii Valenciennes in Cuvier \& Valenciennes, 1840; P. jivaro Eigenmann \& Pearson in Eigenmann \& Allen, 1942; $P$. microstoma Steindachner, 1877; P. ornatus Kner, 1858; e 
P. pictus Steindachner, 1877 (LundBerg \& LitTMAnN, 2003).

Durante a análise do material de Pimelodus da região de Santarém, médio e baixo rio Amazonas, encontramos uma nova espécie do gênero, pertencente ao grupo-maculatus, a qual descrevemos aqui.

\section{MATERIAL E MÉTODOS}

Os espécimes examinados pertencem ao Museu de Ciências e Tecnologia, Pontifícia Universidade Católica do Rio Grande do Sul, Porto Alegre (MCP) e Museu de Zoologia da Universidade de São Paulo, São Paulo (MZUSP). As medidas e as contagens foram realizadas conforme LundBerg \& McDADE (1986) e LundBERG et al. (1991a) e as modificações feitas por LUNDBERg \& PARISI (2002), exceto para o comprimento pré-dorsal, que segue LUNDBERG \& MCDADE (1986). As mensurações foram tomadas em linha reta diretamente sobre o corpo do exemplar, com paquímetro digital, e registradas com a precisão de décimo de milímetro. Sempre que possível as medidas foram realizadas no lado esquerdo do corpo.

A análise osteológica e as contagens de vértebras foram feitas em um espécime diafanizado e corado (c\&d) preparado segundo a técnica de TAYLOR \& VAN DYKE (1985). A nomenclatura dos elementos osteológicos segue LundBerg \& McDade (1986) e LundBerg et al. (1991b).

Na descrição, o número de exemplares examinados foi apresentado entre parênteses, e o valor no holótipo, entre colchetes. Na lista de material examinado, os dados das localidades estão dispostos de norte a sul e de oeste a leste na seguinte ordem: país, estado e dados de coleta, seguidos pelo número de exemplares analisados (entre parênteses), número total de exemplares do lote, maior e menor comprimento padrão analisados no lote seguidos pelo acrônomio e número de catálogo, entre parênteses.

\section{Pimelodus tetramerus sp. nov.}

(Figs. 1 - 4)

Material-tipo. Holótipo, BRASIL, Tocantins: rio Palmeiras, drenagem do rio Tocantins, Ponte Alta do Bom Jesus, $\left(12^{\circ} 15^{\prime} 43^{\prime \prime} \mathrm{S}\right.$ 46 $\left.53^{\circ} 36^{\prime \prime W}\right), 112,9 \mathrm{~mm}$, (MZUSP 85809). Parátipos, BRASIL, Pará: rio Tapajós, entre Itaituba e São Luís $\left(04^{\circ} 27^{\prime} \mathrm{S} 56^{\circ} 15^{\prime} \mathrm{W}\right), 2$, 152,6 e 153,6 mm (MZUSP 34514); Marabá, rio Tocantins $\left(05^{\circ} 22^{\prime} \mathrm{S} 4^{\circ} 07^{\prime} \mathrm{W}\right)$, (4) 10, 74,8-120,1 mm (MZUSP 24620); Tocantins: mesma localidade do holótipo, 2, 89,9 e 124,5 mm (MCP 35830); mesma localidade de coleta do holótipo, 1, 164,6 mm (MZUSP 83811); mesma localidade do holótipo, (2) 18, 112,3 e 113,8 mm (c\&d) (MUZSP 83813); mesma localidade de coleta do holótipo, 3, 112,9-172.1 mm (MZUSP 84004); Mato Grosso: rio Teles Pires, Porto do Alcindo $\left(09^{\circ} 59^{\prime} 25^{\prime \prime} \mathrm{S} 55^{\circ} 33^{\prime} 48^{\prime \prime} \mathrm{W}\right), 1$, $101,9 \mathrm{~mm}$ (MZUSP 85810); rio Araguaia, lago marginal ao rio das Mortes (1249'25"S 5101'13”W), 1, 67,3 mm (MCP 35829); rio Tapajós, rio Teles Pires, fazenda do Antônio Bezerra (14²6'94"S $\left.54^{\circ} 36^{\prime} 75^{\prime \prime} \mathrm{W}\right), 3,108,3-137,3 \mathrm{~mm}$ (MZUSP 63045); rio Araguaia, rio das Mortes em Capitariguara, a jusante de Nova Xavantina

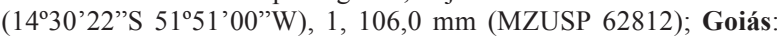

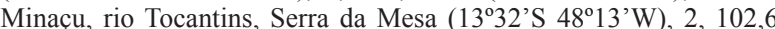
e 107,0 mm (MZUSP 54085); rio Tocantins, $3 \mathrm{~km}$ a jusante do eixo da barragem da Serra da Mesa (13 $\left.3^{\circ} 50^{\prime} 51^{\prime \prime} S 48^{\circ} 16^{\prime} 60^{\prime \prime} \mathrm{W}\right), 2$, 97,2 e 98,7 mm (MCP 35828); rio Tocantins, Flores de Goiás, clube de pesca no rio Paraná (14 $\left.26^{\circ} \mathrm{S} 47^{\circ} 03^{\prime} \mathrm{W}\right), 2,115,5$ e 119,9 mm (MZUSP 40439).

Diagnose. A morfologia tripartida dos foramens trigeminofascial insere a nova espécie no grupomaculatus. Pimelodus tetramerus sp. nov. difere-se das demais espécies deste grupo e de todas as outras do gênero, por sua peculiar coloração que consiste de quatro faixas escuras sobre os lados do corpo. A primeira, mais dorsal, iniciando-se logo após o final da placa pré-dorsal e terminando no final da base da nadadeira adiposa. A segunda, mais larga do que a primeira, logo acima da linha lateral, percorre desde a borda lateral do processo supra-occipital até a base da nadadeira caudal. Essas duas faixas sofrem fragmentação em suas regiões anteriores, formando máculas ou pintas que podem unir-se uma às outras constituindo desenhos vermiculados. Tais máculas e/ou padrão vermiculado se distribui sobre a região pré-dorsal e cabeça. A terceira faixa, tão larga quanto a segunda, logo abaixo da linha lateral, inicia junto à região superior do processo cleitral e estende-se até a base da nadadeira caudal e a quarta, de menor comprimento, inicia logo abaixo do processo cleitral e finaliza próximo à axila da nadadeira pélvica. Além disso, constituem caracteres diagnósticos da nova espécie: (1) a estreita listra clara sobre a linha lateral, com aproximadamente $1 / 3$ da largura da terceira faixa escura, e (2) uma barra escura percorrendo longitudinalmente o lobo ventral da nadadeira caudal. Pimelodus blochii "variedade B" de EIGENMAnN (1912), assemelha-se a $P$. tetramerus pelo padrão geral do colorido. Entretanto, difere daquela por possuir estreita largura interorbital (20,1 a $32,2 \%$ no comprimento da cabeça, média $=24,7 \%$ versus, 25,6 a $34,3 \%$ média $=30,8 \%$ ) e nadadeira adiposa mais longa ( 21,0 a $28,1 \%$ no comprimento padrão, média $=23,8 \%$ versus 18,6 a $24,9 \%$ média $=21,2 \%$ ).

Descrição. Dados morfométricos na Tabela I. Corpo mais alto que largo; perfil pré-dorsal convexo da extremidade do focinho até a placa nucal; levemente côncavo do último raio da nadadeira dorsal até o início da nadadeira adiposa; declinado daí até o pedúnculo caudal; perfil ventral reto da extremidade da mandíbula até a região anterior do istmo, convexo na região abdominal e reto daí até o início da nadadeira anal, de onde se inclina até o pedúnculo caudal. Pedúnculo caudal com perfil dorsal côncavo e ventral convexo.

Cabeça cônica, coberta por pele; superfície com granulação óssea escassa, pouco nítida. Olhos grandes, com diâmetro horizontal sempre maior que o diâmetro vertical e, na maioria das vezes, maior que a largura interorbital. Processo supra-occipital arredondado lateralmente; amplo na base e se estreitando em direção à placa nucal, onde suas extremidades laterais curvam-se para formar uma extremidade com aspecto circular em vista frontal, quase sempre tocando a primeira placa nucal. Placa nucal 1 triangular, coberta por pele fina, superfície ornamentada com escassa granulação óssea; placa nucal 2 menos ornamentada do que a placa 1 , dividida lateralmente na base do primeiro espinho da nadadeira dorsal.

Boca ampla, com abertura maior que a largura interorbital. Lábios dorsais mais carnosos que os ventrais. Dentes cônicos e finos; pré-maxila com 6 a 9 fileiras dispostas aleatoriamente em uma banda 
Tabela I. Dados morfométricos de Pimelodus tetramerus sp. nov. conforme a drenagem (H, Holótipo MZUSP 85809; n, número de exemplares medidos). Cálculos incluem o holótipo.

\begin{tabular}{|c|c|c|c|c|c|c|c|c|c|}
\hline & & \multicolumn{4}{|c|}{ Tocantins } & \multicolumn{4}{|c|}{ Tapajós } \\
\hline & $\mathrm{H}$ & $\mathrm{n}$ & Média & Menor & Maior & $\mathrm{n}$ & Média & Menor & Maior \\
\hline Comprimento padrão (mm) & 112,9 & 21 & 110,7 & 67,3 & 172,1 & 6 & 131,6 & 101,9 & 153,6 \\
\hline \multicolumn{10}{|c|}{ Porcentagens do comprimento padrão } \\
\hline Compr. pré-dorsal & 38,4 & 21 & 39,7 & 37,3 & 40,9 & 6 & 41,6 & 40,9 & 42,1 \\
\hline Compr. pré-anal & 72,4 & 21 & 74,4 & 72,2 & 77,6 & 6 & 76,2 & 74,7 & 77,5 \\
\hline Compr. cabeça & 28,0 & 20 & 27,6 & 25,9 & 29,3 & 6 & 29,0 & 28,2 & 29,9 \\
\hline Compr. pedúnculo caudal & 16,7 & 21 & 15,2 & 11,8 & 17,4 & 6 & 13,9 & 12,8 & 15,2 \\
\hline Altura pedúnculo caudal & 8,7 & 21 & 8,5 & 7,8 & 9,2 & 6 & 8,1 & 7,8 & 8,4 \\
\hline Compr. base adiposa & 23,6 & 21 & 24,2 & 21,0 & 28,1 & 6 & 22,2 & 21,3 & 23,1 \\
\hline Altura da adiposa & 7,4 & 21 & 6,7 & 5,8 & 7,6 & 6 & 6,3 & 5,9 & 6,8 \\
\hline Espaço dorsal-adiposa & 13,0 & 21 & 12,8 & 10,3 & 14,5 & 6 & 13,5 & 11,7 & 14,8 \\
\hline Base anal & 12,2 & 21 & 11,9 & 11,0 & 13,5 & 6 & 11,6 & 10,5 & 12,2 \\
\hline Compr. raio anal & 15,7 & 21 & 15,8 & 14,4 & 17,3 & 4 & 15,6 & 14,3 & 16,5 \\
\hline Compr. raio pélvico & 18,0 & 21 & 17,1 & 15,2 & 19,2 & 6 & 16,7 & 15,8 & 18,6 \\
\hline Compr. raio dorsal & 27,3 & 18 & 26,5 & 23,3 & 29,3 & 5 & 24,2 & 21,8 & 27,9 \\
\hline Distância urogenital-anal & 11,0 & 21 & 12,6 & 11,0 & 14,8 & 6 & 12,2 & 10,9 & 13,4 \\
\hline Compr. espinho dorsal & 25,2 & 18 & 25,4 & 21,8 & 30,5 & 4 & 24,5 & 20,6 & 27,9 \\
\hline Compr. espinho peitoral & 21,2 & 19 & 19,9 & 17,8 & 21,7 & 4 & 20,1 & 18,4 & 21,2 \\
\hline Altura do corpo & 25,9 & 21 & 24,2 & 20,9 & 27,5 & 6 & 22,7 & 21,0 & 24,2 \\
\hline Largura do corpo & 18,3 & 21 & 18,3 & 16,9 & 19,7 & 6 & 18,9 & 18,5 & 19,4 \\
\hline Compr. cleitral posterior & 12,9 & 21 & 13,6 & 12,1 & 14,8 & 6 & 13,9 & 13,1 & 14,5 \\
\hline \multicolumn{10}{|c|}{ Porcentagens do comprimento da cabeça } \\
\hline Altura da cabeça & 64,6 & 20 & 66,3 & 61,0 & 74,3 & 6 & 62,3 & 59,8 & 66,3 \\
\hline Largura interorbital & 20,9 & 20 & 24,5 & 20,1 & 32,2 & 6 & 25,3 & 22,3 & 30,9 \\
\hline Compr. focinho & 44,6 & 20 & 46,0 & 43,2 & 49,5 & 6 & 46,3 & 45,1 & 47,5 \\
\hline Compr. internasal & 15,2 & 20 & 15,3 & 14,5 & 16,2 & 6 & 14,5 & 13,7 & 15,3 \\
\hline Largura internasal anterior & 14,6 & 20 & 14,7 & 13,0 & 18,2 & 6 & 13,9 & 12,2 & 17,2 \\
\hline Largura internasal posterior & 20,3 & 20 & 20,1 & 18,7 & 21,6 & 6 & 18,9 & 17,6 & 19,9 \\
\hline Diâmetro do olho & 31,0 & 20 & 30,9 & 28,5 & 33,3 & 6 & 28,7 & 27,1 & 30,3 \\
\hline Abertura da boca & 32,0 & 20 & 33,1 & 29,7 & 38,0 & 6 & 30,8 & 27,3 & 36,6 \\
\hline Largura do supra-occipital & 22,8 & 20 & 23,8 & 19,9 & 26,2 & 6 & 23,2 & 21,3 & 25,0 \\
\hline Compr. supra-occipital & 32,3 & 20 & 34,7 & 30,4 & 41,6 & 6 & 36,0 & 33,4 & 40,0 \\
\hline
\end{tabular}

ininterrupta que se afila posteriormente, terminando em uma extremidade com aspecto circular, aproximadamente da mesma largura da banda de dentes do dentário; dentes do dentário em uma banda com cerca de 6 a 8 fileiras de dentes cônicos, interrompida ao longo da sínfise mandibular. Dentes no vômer presentes ou ausentes, dentes no osso pterigóide ausentes.

Narinas anteriores tubulares localizadas na região ântero-dorsal do focinho, pouco antes da vertical que passa pela margem anterior da base dos barbilhões maxilares. Narinas posteriores com uma conspícua aba e situadas mais próximas às narinas anteriores que à borda anterior da órbita.

Barbilhões lisos, levemente achatados, variando o comprimento ontogeneticamente. Barbilhões maxilares geralmente estendendo-se além da extremidade posterior da nadadeira adiposa, podendo ultrapassar os lobos da nadadeira caudal em exemplares muito jovens (menores que $80,0 \mathrm{~mm} \mathrm{CP}$ ), porém, não ultrapassando a extremidade dos raios medianos da nadadeira caudal em exemplares acima de 100,0 mm CP; barbilhões mentonianos externos alcançando, ou próximos, a base da nadadeira ventral em adultos e podendo ultrapassá-la em jovens (menores que 100,0 mm CP); em adultos, barbilhões mentonianos internos atingindo entre a base da nadadeira peitoral e a metade de seu menor raio, e podendo ir pouco além da metade deste raio em jovens.

Fontanela anterior ampla, com início próximo à vertical que passa entre as narinas posteriores e final pouco antes da vertical que passa pela margem posterior das órbitas. Fontanela posterior ausente; quando presente, reduzida a um pequeno orifício localizado na base do osso supra-occipital. Opérculo com superfície estriada.

Rastros branquiais finos e longos, os maiores às vezes apenas pouco menores que os filamentos branquiais; os menores pouco maiores que a metade dos filamentos, 4-7+12-17 rastros no primeiro arco branquial $($ moda $=5+15, n=26)[5+16]$. Membranas branquiostégias unidas um pouco antes da vertical que passa pela margem anterior da órbita, 8 raios [8].

Linha lateral iniciando-se junto ao supracleitro e declinando em direção à base dos raios medianos da nadadeira caudal; ossículos tubulares que envolvem a linha lateral expostos externamente.

Nadadeira dorsal com II +6 raios $(\operatorname{moda}=6, n=26)$ [6], sua margem distal declinando em uma linha reta em um ângulo de aproximadamente $45^{\circ} \mathrm{com}$ a nadadeira 

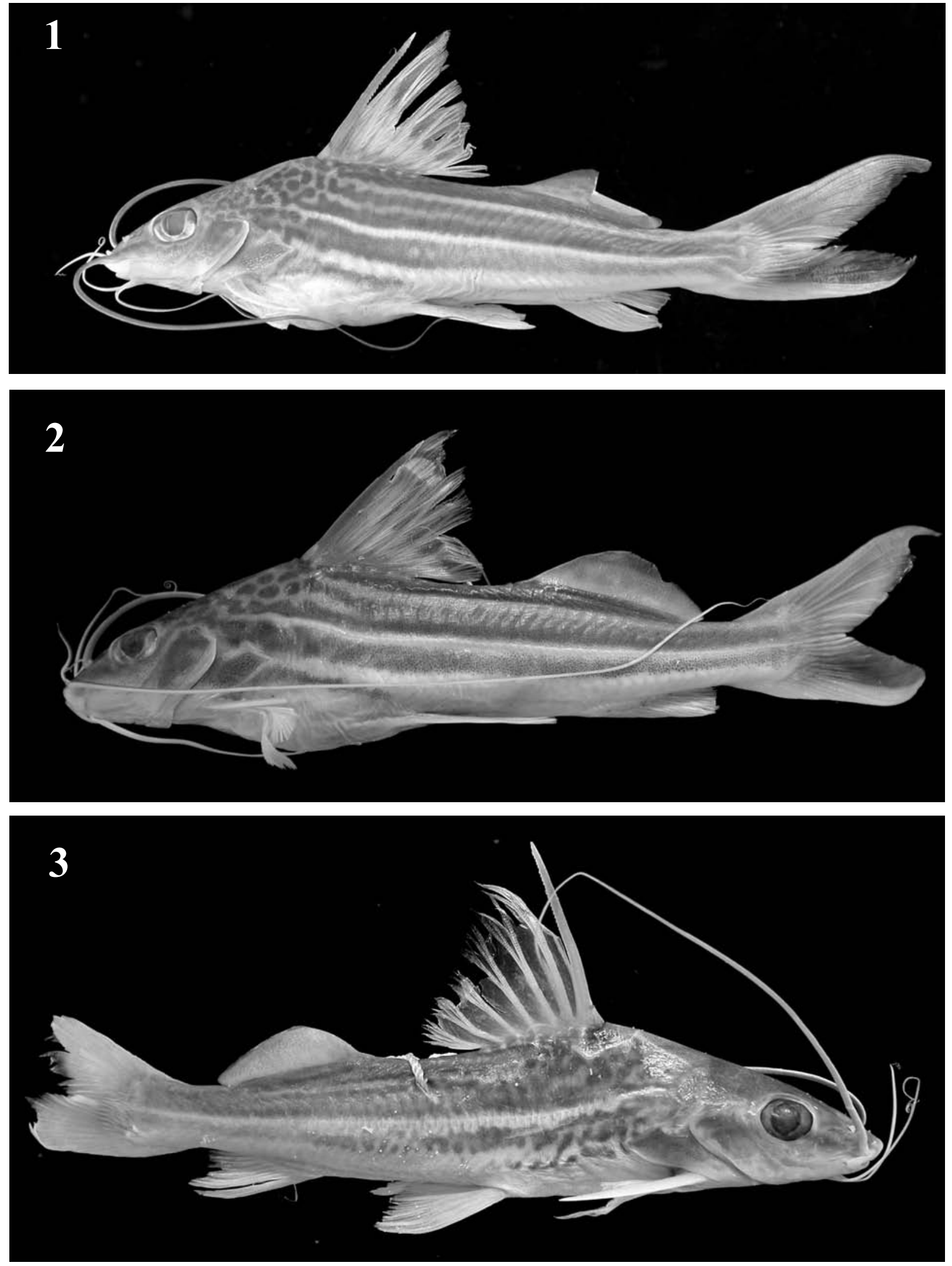

Figs. 1-3. Pimelodus tetramerus sp. nov., rio Tocantins, Tocantins: 1, Holótipo MZUSP 85809, 112,9 mm CP; 2, Parátipo, MCP 35830 , 89,9 mm CP; 3, Parátipo, MZUSP 34514, 152,6 mm CP, rio Tapajós, Pará. 
estendida; o último raio pouco menor que a metade do primeiro. Primeiro espinho muito pequeno e triangular em vista frontal. Segundo espinho grande, robusto, continuando-se em um filamento que se estende além do primeiro raio ramificado da nadadeira; margem anterior lisa ou com até quatro pequenas serrilhas; margem posterior $\operatorname{com} 22$ a $24(\operatorname{moda}=22, \mathrm{n}=4)$ [22] serrilhas voltadas para baixo, ausentes apenas no primeiro quarto do espinho. Nadadeira adiposa moderadamente longa e alta, com comprimento maior que a distância entre o fim da base da nadadeira dorsal e o início de sua base; perfil dorsal convexo e arredondado em sua extremidade posterior; maior altura situada aproximadamente na vertical que passa pela base do primeiro raio ramificado da nadadeira anal.

Nadadeira peitoral, $\mathrm{I}+8-11$ (moda $=9, \mathrm{n}=25)$ [9], com um espinho robusto; sua margem anterior convexa, com pequenas serras em exemplares mais jovens e pequenas granulações ósseas em exemplares maiores, ambos situadas na primeira metade do espinho; margem posterior do espinho com 19 a 30 (moda=28, n=10) [30] serrilhas, distribuídas em toda sua extensão. Nadadeira pélvica com $\mathrm{i}+5$ raios $(\operatorname{moda}=5, \mathrm{n}=26)$ [5], inserida aproximadamente na vertical que passa pelo último espinho da nadadeira dorsal; quando adpressa, alcança aproximadamente dois terços do caminho entre sua base e o início da anal. Nadadeira anal com iv-vi $+7-9$ raios $($ moda $=\mathrm{v}+8, \mathrm{n}=25)$ [vi+7]; quando recolhida, alcança ao menos os raios procorrentes da caudal; o último raio simples e os dois primeiros raios ramificados geralmente maiores que os demais. Nadadeira caudal profundamente furcada, $i+7+8+i$ raios $(\operatorname{moda}=\mathrm{i}+7+8+\mathrm{i}, \mathrm{n}=26)[\mathrm{i}+7+8+\mathrm{i}]$, lobo dorsal levemente maior que o ventral, 16 raios procorrentes caudais no ramo dorsal e 20 no ventral, em um exemplar diafanizado e corado.

Vértebras 41: 15 pré-caudais, 26 caudais, em um exemplar diafanizado e corado.
Colorido em álcool. Quatro faixas escuras sobre os flancos, sendo duas acima e duas abaixo da linha lateral; a primeira, mais dorsal, inicia logo após o final da placa pré-dorsal e termina no final da base da nadadeira adiposa; a segunda, mais larga que a primeira, logo acima da linha lateral, percorre desde a borda lateral do processo supraoccipital até a base da nadadeira caudal; a terceira, também larga, logo abaixo da linha lateral, inicia junto à região superior do processo cleitral e estende-se até a base da nadadeira caudal e a quarta, de menor comprimento e que pode estar fraca ou ausente, inicia logo abaixo do processo cleitral e finaliza próximo à nadadeira pélvica. As duas faixas escuras acima da linha lateral sofrem fragmentação em suas regiões anteriores, formando máculas que podem unir-se uma às outras constituindo desenhos vermiculados (Figs. 1, 2); tais máculas e/ou padrão vermiculado se distribui sobre a região pré-dorsal e cabeça. Em conseqüência de tal fragmentação, pode ocorrer uma estreita e tênue divisão da segunda faixa que se estende, algumas vezes, até a vertical que passa logo à frente da nadadeira adiposa. Provavelmente a fragmentação das duas primeiras faixas ocorra mais amplamente com o crescimento, quando ambas se tornam fragmentadas em toda a extensão, inclusive entre a terceira e quarta faixas (Fig. 3). Uma listra clara percorre toda a extensão da linha lateral, com aproximadamente $1 / 3$ da largura da terceira faixa escura. Nadadeira caudal com uma conspícua barra longitudinal escura no lobo ventral; lobo dorsal algo escurecido. As outras nadadeiras hialinas a levemente escurecidas. Ventre claro, sem manchas. (Fig. 4).

Distribuição. Rios Tocantins e Tapajós, Brasil

Etimologia. O nome específico tetramerus, do grego tetra + meros, significa em quatro partes, e é dado em referência às quatro faixas escuras na lateral do corpo.

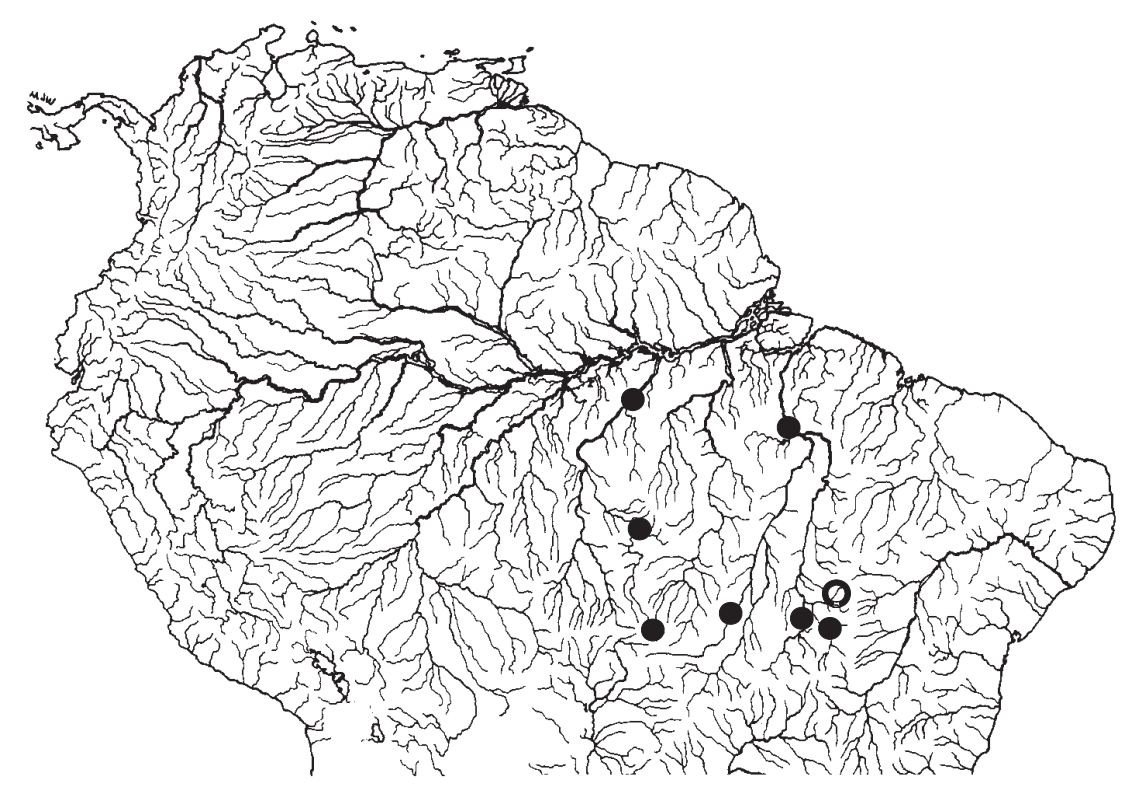

Fig. 4. Distribuição geográfica de Pimelodus tetramerus sp. nov. Círculo aberto indica a localidade-tipo. 
Material comparativo examinado. Seguindo o nome específico estão: número de catálogo, número de espécimes, Alc, álcool; F, fotografia; Cc, diafanizado e corado, e procedência.

Pimelodus absconditus MCP 13392, 2, 129,3 e 133,7 mm, Alc, Brasil, rio Uruguai; MCP 12515, 1, 125,0 mm, Alc, Brasil, rio Uruguai. Pimelodus albicans MCP 19248, 4, 139,9-199,6 mm, Alc, Argentina, rio Paraná; Pimelodus albofasciatus RMNH 26156, 1, F, Sipaliwini, Suriname; Pimelodus altissimus MZUSP 24588, 1, 148,9 mm, Alc, Brasil, rio Purus; Pimelodus cf. argenteus MCP 15811, 4, 53,5-166,5 mm, Alc, Brasil, rio Paraguai; Pimelodus atrobrunneus MCP 19678, Holótipo, 131,5 mm, Alc, Brasil, rio Uruguai; Pimelodus blochii "variedade A" MCP 24486, 2, 86,2 e 85,2 mm, Alc, Brasil, rio Amazonas; MZUSP 34513, 2, 151,3 e 154,0 mm, Alc, Brasil, rio Xingu; MZUSP 54558, (5) 14, 75,1-117,4 mm, Alc, Brasil, rio Tapajós; Pimelodus blochii "variedade B" MCP 35831, 24, 94,0132,7 mm, Alc, Brasil, rio Tapajós; MCP 34152, 1 (3), 84,7 mm, Cc, Brasil, rio Tocantins; MZUSP 33314, (3) 6, 107,1175,8 mm, Alc, Brasil, rio Negro; MZUSP 40439, 2, 115,5 e 120,0 mm, Alc, Brasil; MZUSP 54085, 2, 102,6 e 107,0 mm, Alc, Brasil; MZUSP 52307, 2, 141,4 e 162,9 mm, Alc, Brasil, rio Araguaia; MZUSP 53747, 2, 82,6 e 139,5 mm, Alc, Brasil, rio Araguaia; MZUSP 34514, 2, 152,6 e 153,6 mm, Alc, Brasil, rio Tapajós; MZUSP 30738, 3, 87,3-101,1 mm, Alc, Brasil, rio Tapajós; MZUSP 57742, 2, 86,2 e 89,5 mm, Alc, Brasil, rio Tapajós; MZUSP 57943, 3, 99,2-114,8 mm, Alc, Brasil, rio Trombetas; MZUSP 24215, 3, 84,4-101,3 mm, Alc, Brasil, rio Tocantins; Pimelodus fur MCP 14051, 5 , 84,8-111,3 mm, Alc, Brasil, São Francisco; Pimelodus jivaro MZUSP 56729, 1, 93,3 mm, Cc, Brasil, rio Xingu; MZUSP 57672, 2, 92,5 e 90,9 mm, Alc, Brasil, rio Trombetas; Pimelodus heraldoi MZUSP 22713, Holótipo, 179,0 mm, F, Brasil, rio Mogi Guaçu; Pimelodus maculatus MCP 19249, 3, 56,7-102,0 mm, Alc - Cc, Argentina, rio Paraná; MCP 13235, 1, 167,7 mm, Alc, Brasil, rio Uruguai; Pimelodus mysteriosus MLP 9191, Holótipo, 103,0 mm, F, Argentina, arroio Anselmo; Pimelodus microstoma NMW 45824, 2, 125,8 e 140,0 mm, Alc, Brasil; Pimelodus ornatus MCP 28870, 1, 90,7 mm, Alc, Brasil, rio Purus; MCP 15898, 1, 152,7 mm, Alc, Brasil, rio Tocantins; MZUSP 34480, 2, 125,6 e 130,6 mm, Alc, Brasil, rio Tapajós; Pimelodus paranaensis MZUSP 23089, Holótipo, 235,0 mm, F, Brasil, rio Paraná; Pimelodus pictus MCP 29853, 1, 72,0 mm, Alc, Brasil, rio Solimões; MZUSP 25976, 1, 67,4 mm, Alc, Peru, rio Ucayali.

\section{DISCUSSÃO}

Existe uma grande diversidade de colorido entre as espécies de Pimelodus (sensu lato) na bacia Amazônica. Dentre estes padrões, os apresentados por P. ornatus e P. pictus são os mais característicos. Pimelodus ornatus possui uma grande mancha na nadadeira dorsal e duas faixas escuras largas nos lados do corpo, além de possuir a pré-maxila projetada, com ampla placa de dentes. Pimelodus pictus apresenta algumas manchas grandes distribuídas sobre os flancos e nadadeiras, e as narinas posteriores são muito próximas às órbitas. Estes caracteres distinguem facilmente estas duas espécies de P. tetramerus.
Pimelodus altissimus e P. jivaro possuem coloração uniforme variando de cinza-azulada a cinzaescuro. Além disso, estas espécies possuem a nadadeira adiposa longa, preenchendo quase todo o espaço entre o fim da base da nadadeira dorsal e o início do pedúnculo caudal em $P$. altissimus, e pouco menor em $P$. jivaro.

Conforme descrito por EIGENMANN (1912), existem duas "variedades" de Pimelodus blochii na bacia Amazônica. A "variedade A", com corpo cinzento, sem pintas ou faixas, e a "variedade B", com quatro faixas pretas sobre os lados do corpo, sendo que a quarta pode estar ausente ou fragmentada em pintas.

Mees (1974) descreveu Pimelodus albofasciatus a partir de espécimes do Suriname. Ao comparar Pimelodus blochii "variedade B" de EigenMAnN (1912) com sua espécie, considerou ambas extremamente similares, porém $P$. albofasciatus diferindo por possuir olhos menores $(0,8$ a 1,6 vezes na largura interorbital versus 0,7 a 1,0 ) e pelo comprimento do espinho dorsal (maior ou igual ao comprimento da cabeça versus menor ou igual).

Apesar da coloração similar àquela apresentada por P. tetramerus, $P$. albofasciatus, assim como P. blochii "variedade B" de EIGENMANN (1912), não possui a barra preta na nadadeira caudal e nem as máculas escuras, ou vermiculações, na cabeça e na região látero-dorsal do corpo.

Com relação ao sistema Tocantins-Araguaia, até o momento, há o registro de duas espécies de Pimelodus: P. blochii e P. ornatus (SAntos et al., 1984). Analisando o material colecionado desta bacia, observamos a ocorrência de P. blochii "variedades A e B" de EIGENMANN (1912), além de uma outra espécie ainda não descrita que, apesar de possuir uma barra preta no lobo ventral da nadadeira caudal, difere de Pimelodus tetramerus por possuir pequenas pontuações escuras distribuídas aleatoriamente sobre os flancos (espécie nova em descrição por Flávio Bockmann, comun. pess.).

Pimelodus microstoma foi descrita das bacias dos rios Amazonas e Mogi-Guaçu, rio Paraná superior (localidade tipo: von Irisanda [sic], Rio Branco und Barra do Rio Negro) por Steindachner (1877). No entanto, análises preliminares indicam que esta espécie provavelmente não ocorra no sistema amazônico. De qualquer forma, P. microstoma possui coloração uniforme, com poucas e pequenas pintas escuras nos lados, as quais podem ser fracas ou mesmo ausentes, além de não apresentar a barra preta no lobo ventral da nadadeira caudal, portanto distinta de $P$. tetramerus.

Além das espécies formalmente descritas e da nova espécie da bacia do rio Tocantins citada acima, verificamos a existência de pelo menos duas outras ainda não descritas na bacia Amazônica que, assim como $P$. tetramerus, possuem quatro faixas sobre os flancos e uma barra escura no lobo ventral da nadadeira caudal. Estas, porém, não possuem as máculas escuras, ou vermiculações, na cabeça e na região látero-dorsal do corpo e a listra clara sobre a linha lateral é larga (cerca da metade, ou mais, da largura da terceira faixa escura).

LUNDBERG et al. (1991b) observaram que muitos Siluriformes possuem um padrão relativamente simples de foramens do nervo trigeminofacial no pró-ótico e ptero-esfenóide em contraste a outros teleósteos. Este padrão simples estaria presente na maioria dos 
Siluriformes, e seria um único e grande forâmen no limite pró-ótico e ptero-esfenóide, ou raramente apenas no próótico, para as maiores ramificações do quinto e sétimo nervos craniais. Tal padrão é a condição primitiva de pimelodídeos, podendo ser encontrada também em heptapterídeos e pseudopimelodídeos. Em uns poucos Pimelodidae existiria uma subdivisão do forâmen trigeminofacial em forâmen facial e trigeminal. Dentre as espécies de Pimelodus assinaladas para a Amazônia, segundo LundBerg et al. (1991b) e LundBerg \& PARISI (2002) existiria um forâmen adicional, o qual seria formado a partir da divisão do forâmen trigeminal, em $P$. blochii, $P$. jivaro e P. pictus ( $P$. albofasciatus e P. microstoma, citadas para a Amazônia, ainda não foram examinadas quanto ao caráter). Compartilham ainda do mesmo caráter, mas não assinaladas para a Amazônia, segundo os mesmos autores, P. maculatus, P. coprophagus Schultz, 1944 e $P$. grosskopfi Steindachner, 1879. Nessas, há três foramens separados, um para cada nervo maxilar, mandibular e hiomandibular. Pimelodus tetramerus compartilha deste suposto caráter derivado indicando que provavelmente esteja relacionada com essas espécies. Pimelodus ornatus e P. altissimus possuem a condição primitiva do caráter (LuNDBERG et al., 1991b).

Embora a proposição de hipóteses de relações de $P$. tetramerus não faça parte do escopo deste trabalho, registramos que $P$. tetramerus possui o ramo posterior do processo dorsal do pré-maxilar parcialmente articulado por cartilagem com a margem látero-anterior do mesetmóide. Segundo Azpelicueta (2001) essa é a condição primitiva, também presente em $P$. maculatus, $P$. blochii, P. ortmani, P. mysteriosus e P. argenteus. Outra condição provavelmente primitiva (AzPelicueta, 2001:198) presente em P. tetramerus é a forma arredondada do canto póstero-lateral do mesetmóide (versus em ângulo reto em $P$. heraldoi). Por outro lado, a presença de uma desenvolvida crista no faringobranquial 3, presente somente em $P$. heraldoi (AZPELICUETA, 2001: fig. 5 a), pode indicar relação entre essa espécie e $P$. tetramerus. Finalmente, a condição exposta do centrum da vértebra 7, presente em P. tetramerus, foi relacionada à variação ontogenética (cf. AzPelicueta, 2001). A condição não-exposta do centrum da vértebra 7 , é dada por LUNDBERG et al. (1991b) como sinapomorfia para definir um clado monofilético onde está o gênero Pimelodus.

Agradecimentos. A Osvaldo Oyakawa e José Figueiredo (MZUSP) pelo empréstimo de material e ao apoio técnico dado ao primeiro autor durante o exame da coleção; John Lundberg (Academy of Natural Science of Philadelphia) forneceu dados inéditos e fotos de pimelodídeos sob seu estudo; Sidcley Matos (Faculdades Integradas do Tapajós, FIT) providenciou infraestrutura durante as coletas realizadas na região de Santarém; a United States National Science Foundation, que fomentou à J. G. Lundberg (DEB 0089612) recursos para viagens de campo ao autor-sênior. Roberto Reis (PUCRS) auxiliou no Abstract. Dois consultores anônimos deram importante contribuição à forma final do trabalho.

\section{REFERÊNCIAS BIBLIOGRÁFICAS}

Azpelicueta, M. DE las M. 2001. A new species of Pimelodus (Siluriformes: Pimelodidae) from the upper Paraná basin, Brazil. Ichthyological Explorations Freshwaters 12(3):193-200.

DE PinnA, M. C. C. DE. 1998. Phylogenetic relationships of neotropical Siluriformes: historical overview and synthesis of hypoteses. In: Malabarba, L. R.; Reis, R. E.; VAri, R. P.; Lucena, Z. M. S. \& Lucena, C. A. S. eds. Phylogeny and Classification of Neotropical Fishes. Museu de Ciências e Tecnologia, PUCRS, Porto Alegre. p. 379-330.

Eigenmann, C. H. 1912. The fresh-water fishes of British Guiana, including a study of the ecological grouping of species, and the relation of the fauna of the plateou to that of the lowlands. Memoirs of the Carnegie Museum 5(67):1578, pls. $1-103$.

Lundberg, J. G. \& Littmann, M. W. 2003. Family Pimelodidae (Long-whiskered catfishes). In: Reis, R. E.; Kullander, S. O. \& Ferraris, JR., C. J. eds. Check List of the Freshwater Fishes of South and Central America. Edipucrs, Porto Alegre. p. 432-446.

Lundberg, J. G. \& McDade, L. A. 1986. On the South American catfish Brachyrhamdia imitator Myers (Siluriformes, Pimelodidae), with phylogenetic for a large intrafamilial lineage. Notulae Naturae 463:1-24.

Lundberg, J. G. \& PArisi, B. M. 2002. Propimelodus new genus, and redescription of Pimelodus eigenmanni Van der Stigchel 1946, a long-recognized yet poorly-known South American catfish (Pimelodidae: Siluriformes). Proceedings of the Academy of Natural Sciences of Philadelphia 152:75-88.

Lundberg, J. G.; Bornbusch, A. H. \& Mago-Leccia, F. 1991a. Gladioglanis conquistador N. Sp. from Ecuador with diagnoses of the subfamilies Rhamdiinae Bleeker and Pseudopimelodinae N. Sub f. (Siluriformes: Pimelodidae). Copeia 1991(1):190-209.

Lundberg, J. G.; Mago-Leccia, F. \& Nass, P. 1991b. Exalloduntus aguanai, a new genus and species of Pimelodidade (Pisces: Siluriformes) from deep river channels of South América, and delimitation of the subfamily Pimelodinae. Proceedings of the Biological Society of Washington 104(4):840-869.

Mees, G. F. 1974. The Auchenipteridae and Pimelodidae of Suriname (Pisces, Nematognathi). Zoologische Verhandelingen 132:1-256

Reis, R. E.; Kullander, S. O. \& Ferraris, Jr., C. J. eds. 2003. Check List of the Freshwater Fishes of South and Central America. Edipucrs, Porto Alegre. 729p.

Santos, G. M.; Jégu, M. \& de Merona, B. 1984. Catálogo de peixes comerciais do Baixo Rio Tocantins. ELETRONORTE, CNPq e INPA, Brasília. 83p.

Steindachner, F. 1877. Die Süsswasserfische des südöstlichen Brasilien (III). Sitzungsberichte der Kaiserlichen Akademie der Wissenschaften, Mathematisch Naturwissenschaftlichen Classe Wien, Abtheilung 1: Botanik, Zoologie, Anatomie, Geologie und Paläontologie, 74:559-694.

Swarça, A. C.; Caetano, L. G. \& Dias, A. L. 2000. Cytogenetics of species of the families Pimelodidae and Rhamdiidae (Siluriformes). Genetics and Molecular Biology 23(3):589-593.

TAYlor, W. R. \& VAN Dyke, G. C. 1985. Revised procedures for staining and clearing small fishes and other vertebrates for bone and cartilage study. Cybium 9(2):107-119.

Vidal, E. S. \& LucenA, C. A. S. 1999. Pimelodus atrobrunneus, uma nova espécie de pimelodídeo do rio Uruguai superior (Teleostei, Siluriformes, Pimelodidae). Biociências 7(1):121-134

Recebido em dezembro de 2004 . Aceito em junho de 2006. ISSN 0073-4721

Artigo disponível em: www.scielo.br/isz

Iheringia, Sér. Zool., Porto Alegre, 96(3):321-327, 30 de setembro de 2006 